\title{
Cave chamber data modeling and 3D Web visualization
}

\author{
Ivo Silvestre $^{1}$, José I. Rodrigues ${ }^{1,2}$, Mauro Figueiredo ${ }^{1,2}$, Cristina Veiga-Pires ${ }^{1,3}$ \\ ${ }^{1}$ Centro de Investigação Marinha e Ambiental - CIMA \\ ${ }^{2}$ Instituto Superior de Engenharia - Universidade do Algarve \\ ${ }^{3}$ Faculdade de Ciências e Tecnologia - Universidade do Algarve \\ \{imsilvestre,jirodrig,mfiguei,cvpires\}@ualg.pt
}

\begin{abstract}
Underground caves and its specific structures are important for geomorphological studies. In this paper we present tools to identify and map speleothems by surveying cave chambers interiors.

The cave chamber was surveyed using Terrestrial Laser Scanning to acquire point clouds with high level of detail for 3D model generation. This data with 45 million points is useful for either reconstruction, geomorphological studies or virtual visits of caves.

With this point cloud we generated a $3 D$-mesh to represent the surface model of the cave chamber, which is important to study its geomorphological features. A topological structure of the 3D-mesh was also implemented to get an efficient algorithm to help identifying stalactites.

The possibility to publish 3D data on the Web is of particular interest for the geospatial field. For this reason, it was decided to make the cave model available in the Web by developing a 3D graphical interface where users can navigate and interact with the three-dimensional models of the cave. For this Web3D framework it was used X3D, WebGL and X3DOM. Such solution does not require any additional plug-ins or components.
\end{abstract}

Keywords - Cave surveying, TLS, 3D-mesh, MeshLab, X3D, X3DOM

\section{Introduction}

In the context of project SIPCLIP ${ }^{1}$, which is concerned with the global warming, it aims to provide information on past regional climates, to better constrain their complex interactions with global climate and acquire new information to document past climatic conditions in the South-western Iberian Peninsula. This information is based on the analysis of cave speleothems, which are useful records for paleoclimatic reconstruction.

${ }^{1}$ PTDC/AAC-CLI/100916/2008 - Temperature, precipitation regime and soil conditions in Southwestern Iberian Peninsula under a warmer climate - Insight from the past
The present paper describes a framework for the generation of a 3D model and a Web3D visualization of the karst cave Algar do Penico located in the southernmost region of Portugal (Algarve). This model was used by our implemented system to enable the identification and mapping of geomorphological structures inside a cave.

Section 2 briefly presents the surveying process of the cave chambers with high level of detail by using a Terrestrial Laser Scan. Chambers have a lot of irregular surfaces. The obtained point cloud from laser scan presents a high level of data density with about 45 million points.

Identifying geomorphological structures is one of the goals of this research project. Point clouds by itself do not provide enough information about the cave chamber surface. Section 3 presents approaches to generate a 3Dmesh from the point cloud using an open source tool.

In the Section 4 we present algorithms for the study of geomorphological features of caves. Such approaches allow the recognition and positioning of cave stalactites that can provide information on hidden features responsible for cave geomorphology. We also implemented a topological structure for the 3D-mesh to get an efficient algorithm to help determining stalactites.

Web3D sites that include three-dimensional models where users navigate and interact through a 3D graphical interface, are increasingly employed in different domains.

Section 5 presents our approach to make the cave model available in a Web3D site. It provides a 3D graphical interface where users can navigate and interact with the threedimensional model of the cave. To this purpose, it was used X3D, WebGL and X3DOM. Such solution does not require any additional plug-ins or components.

\section{Terrestrial Laser Scanning survey}

The selected cave, known by the local speleologists as Algar do Penico, is located in the southernmost region of Portugal (Algarve), to the west of the city of Loulé. This cave has an extension of about 80 meters, a gap of 14 meters and a depth of approximately 20 meters. It is composed by two chambers connected by a vertical narrow 
gallery of about 5 meters.

In the context of this project and with the objective of mapping the cave and its geomorphological structures for environmental and geological studies, it was decided to survey its interior chambers. Each chamber was surveyed independently due to its morphological characteristics. The work presented in this paper is focused in the main chamber. In order to survey the cave with high levels of data density for close range applications a laser scanner was used. The process of data acquisition in the interior of the cave was a very complex task due to the difficult accesses and the working environment, which has a lot of irregular surfaces.

Terrestrial Laser Scanning is a relatively new technology that offers high resolution surveys. The scanner's pulsed laser ranging device, coupled with beam deflection mechanisms, facilitates rapid acquisition of a huge number of three-dimensional point measurements. One of the great advantage of this surveying method is the high-resolution surface geometry that permits accurate and detailed surface reconstruction and modeling as well as superior visualization relative to existing measurement technologies [1].

The main chamber of the cave Algar do Penico was surveyed with a Terrestrial Laser Scanner (TLS), more specifically, the Leica ScanStation C10 laser scanner system mounted on a tripod. The specifications of this unit include a $360^{\circ}$ horizontal field of view and a maximum of $270^{\circ}$ vertical field of view. The scanner emits pulses of green laser light that sweeps across the chamber surfaces and sends back measurements with precise $x, y$ and $z$ coordinates, each having an associated intensity value and color. The scanner enables point cloud to be captured that correspond to true point positions where the laser pulse hits the object. The point cloud represents the shape and position of scanned surfaces relative to the position of the scanner.

The 3D laser scanner point cloud data was collected with a point spacing of 20 seconds of arc. Distance between closest points may varying according distance from station. Due to the dimensions of the cave chamber the maximum distance between closest points is less than one centimeter. Thus, a total of $45 \times 10^{6}$ point raw data arrays, which is a massive data set.

\section{3D-mesh generation}

Identifying geomorphological structures is one of the goals of this research project. In the previous section, a point cloud with about 45 million points was obtained from laser scan surveys. Point cloud do not provide enough information about the cave chamber surface. For this reason it is necessary to generate a surface model.

Computational representation of surfaces is a widely studied problem, with a vast bibliography of reference.
Surfaces are usually represented by a collection of vertices, edges and faces, known as a polygonal mesh or polygonal soup, defining the shape of a polyhedral object. In the present work, a triangular 3D-mesh was adopted which is more appropriate for visualization. The faces of the mesh consist of triangles, where each face corresponds to a set of three vertices [2].

We used the Meshlab software [3] for the 3D-mesh generation of the cave chamber. MeshLab ${ }^{2}$ is a free and open source software for mesh processing and editing. Furthermore, it works with a huge number of common 3D file formats, such as, PLY, STL, OBJ, 3DS, COLLADA, PTX, PTS, XYZ, ASC, X3D and VRML. There are several algorithms to do the surface reconstruction from point clouds. MeshLab has several methods for surface reconstruction. We explored a ball pivoting variant and Poisson's reconstruction.

The Poisson Surface Reconstruction is based on the observation that the normal field of the boundary of a solid can be interpreted as the gradient of the solid's indicator function. Therefore, given a set of oriented points sampling the boundary of a solid, a 3D-mesh can be obtained by transforming the oriented point samples into a continuous vector field in 3D. This is performed finding a scalar function whose gradients best match the vector field, and extracting the appropriate isosurface [4]. Although it is beyond the scope of this paper a thorough analysis of this algorithm, it is useful to note that the vertices of the faces of these meshes do not coincide with the points of the survey.

The surface obtained with Poisson Surface Reconstruction has a variable detail level depending on the input parameter of the octree depth. The octree depth parameter is the maximum depth of the tree that will be used for the surface reconstruction. Increasing the depth, the surface gets more details. The number of faces and vertices also increases as it is shown in Table 1. This table presents the number of vertices, faces and the file size for several 3Dmeshes generated with octree depths of 10,11, 12 and 13 . Three dimensional meshes are stored in PLY file format. High values of octree depth increase the amount of data from $100 \mathrm{MB}$ to $2.4 \mathrm{~GB}$.

Table 1: 3D-meshes generated from Poisson Surface Reconstruction with octree depths of 10,11, 12 and 13.

\begin{tabular}{|c|c|c|c|}
\hline depth & \# vertices & \# faces & size (MB) \\
\hline 10 & 1316365 & 2630424 & 99 \\
\hline 11 & 5016981 & 10030156 & 396 \\
\hline 12 & 15628234 & 31252894 & 1300 \\
\hline 13 & 28272558 & 56541528 & 2400 \\
\hline
\end{tabular}

A compromise has to be achieved between the com-

\footnotetext{
${ }^{2}$ http://meshlab. sourceforge. net/
} 
plexity of the 3D-mesh, the realistic visualization of the chamber and real time interaction. For these reasons, we used the Poisson Surface Reconstruction method with an octree depth of 11 to generate a 3D-mesh with about 5 million vertices and 10 million faces. This method returned a closed surface even where the point cloud is more sparse. Figure 1 shows the 3D-mesh of the cave chamber, where we can navigate in real time, in a desktop computer with an Intel Core i7 $3.40 \mathrm{GHz}, 8 \mathrm{~GB}$ of memory RAM and a NVIDIA Quadro 4000 graphic card (with 2GB of dedicated memory) using MeshLab. The operating system is the 64-bit Ubuntu 12.04 LTS.

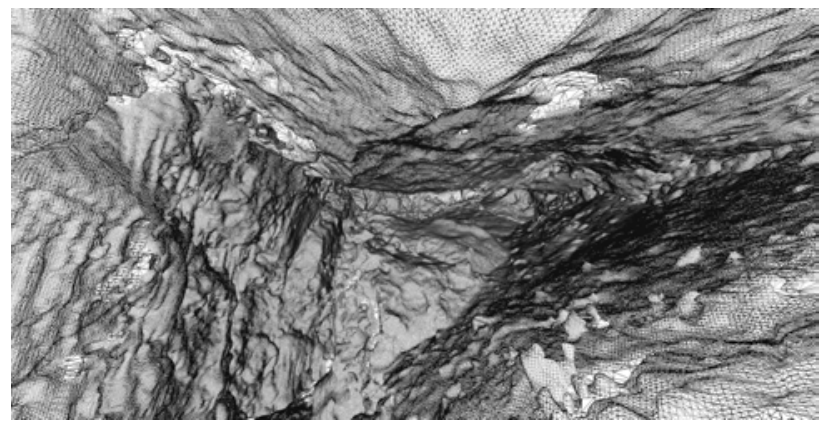

Figure 1: Cave chamber 3D-mesh image obtained with Poisson Surface Reconstruction with a depth of 11.

\section{Identification of geomorphological struc- tures from 3D-mesh}

The speleogenetic studies aimed deciphering the origin of caves and details of specific processes which led to the existing cave structures, by studying cave morphology and its meso- and micro-morphological features. These smaller-scale features are typically not represented on the standard cave map obtained, for example, by electronic distance measurements [1].

One of the main objectives of this work is to identify and map the geomorphological structures existent inside the cave Algar do Penico.

The polygon mesh previously referred (see Section 3) is a mesh that consists of a collection of triangles with no particular ordering and without any kind of topological information associated. This type of structures are the default output format when geometry is exported from a 3D modeling software such as MeshLab, Blender or Maya [5]. In order to identify geomorphological structures, the availability of explicit topological information helps the implementation of efficient algorithms to traverse the huge data surface model of the cave chamber. That can be implemented using graphs.

A graph consists of a finite set of vertices, $V$, and a set of edges, $E$, where each $e \in E$ is a pair of vertices. Given an edge, $e=\{u, v\}$, vertices $u, v \in V$ are adjacents. A path is a sequence of pairwise adjacent vertices without repetition of any vertex except possibly the first and the last which can be the same. When the first and the last vertices of a path are the same, the closed path is also called a cycle. Graphs are often sketched as node-link diagrams in which the vertices are represented as points and the edges as lines. When a graph draw can be embedded in the plane with no edge crossing except at their common vertices the graph is called planar. Let $G=(V, E)$ be a plane graph. The plane regions bounded by the edges of $G$ are called faces.

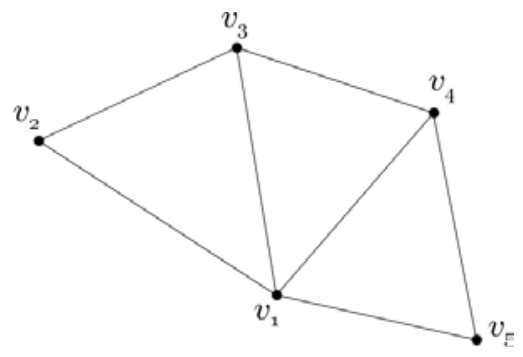

Figure 2: Planar graph with 5 vertices and 7 edges.

Figure 2 presents a planar graph $G$ with 5 vertices and 7 edges and 4 faces ( 3 triangles and the exterior face). Vertex $v_{1}$ is adjacent to all other vertices, $\left\{v_{3}, v_{4}\right\}$ is an edge of $G$, $\left\{v_{1}, v_{2}, v_{3}, v_{4}\right\}$ is a path and $\left\{v_{1}, v_{2}, v_{3}, v_{1}\right\}$ is a face.

There are several data structures to store graphs. For computational purposes, we adopt the adjacency list data structure where for each vertex correspond the list of adjacent vertices.

For the graph in Figure 2, the adjacency list is as follows:

$$
\begin{aligned}
A d j= & \left\{v_{1}:\left[v_{2}, v_{3}, v_{4}, v_{5}\right], v_{2}:\left[v_{1}, v_{3}\right]\right. \\
& \left.v_{3}:\left[v_{1}, v_{2}, v_{4}\right], v_{4}:\left[v_{1}, v_{3}, v_{5}\right], v_{5}:\left[v_{1}, v_{4}\right]\right\}
\end{aligned}
$$

From a 3D-mesh polygonal soup a definition of a graph $G=(V, E)$ is a straight process. The vertices set $V$ is the vertices set of the mesh and edges of $G$ are the edges of the mesh. The advantage of the graph structure is that it includes explicit topological information which allows the implementation of an efficient algorithm to determine local minima in the mesh (see Algorithm 1).

The information of vertex adjacencies was organized with the help of Python ${ }^{3}$ dictionaries. These data types are sometimes found in other languages as associative arrays or hash tables. A Python dictionary is an unordered collection of key-value pairs, with the requirement that the keys are unique. A pair of braces \{\} creates an empty dictionary. Placing a comma-separated list of key-value pairs

\footnotetext{
${ }^{3}$ http: //www.python.org/
} 
within the braces adds initial key-value pairs to the dictionary.The dictionary example with the graph in Figure 2 is presented in (1).

Stalactite extremities correspond to local minima in the 3D-mesh. A local minimum in the 3D-mesh surface is one point $P$ of the mesh such that its $z$ coordinate is less than $z$ coordinates of all points in a chosen neighborhood of $P$.

A local minimum $P$ is a vertex of the $3 \mathrm{D}$-mesh because the faces of this surface are triangles. Thus, given a vertex $v \in V$ to decide if it corresponding point on the 3D-mesh is local minimum it is sufficient to check if its $z$-coordinate is less than the $z$-coordinates of all adjacent vertices of $v$.

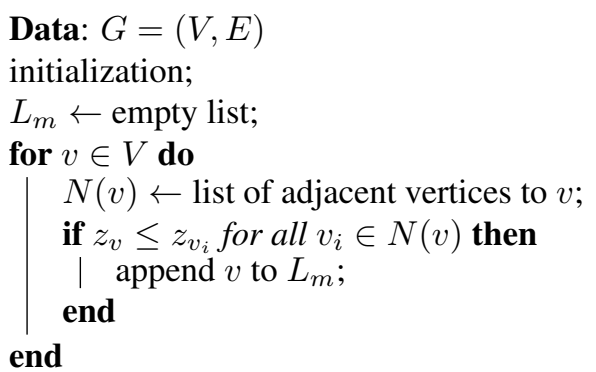

Algorithm 1: Local minimum algorithm.

Regarding the fact that if $u$ is adjacent to $v$ and $z_{u}<z_{v}$ than $v$ is not a local minimum, Algorithm 1 can be implemented without need to check the minimum condition for all vertices of $V$.

Algorithm 1 was used to compute local minima of the chamber using the 3D-mesh model obtained with octree depth of 11 (see Figure 4).

Other important case study from the 3D-mesh, evolving the graph was the extraction of contour lines. Contour lines, also known as contours, are lines that join all points of equal elevation ( $z$-coordinate). Contours are used to denote elevation and depth.

In the context of the present work, contours allow to denote topographic surface of the chamber and are useful structures for water run-off studies. Figure 5 shows $5 \mathrm{~cm}$ equidistant contours of a specific region of stalactites in the interior of Algar do Penico chamber.

\section{3D data visualization on the Web}

As a consequence of advances in computer hardware and internet connection speed, Web3D sites that include three-dimensional models where users navigate and interact through a 3D graphical interface, are increasingly employed in different domains. The possibility to publish 3D data on the Web is of particular interest for the geospatial field. This enables researchers to visualize, navigate and interact with three-dimensional data on a simple Web browser. In addition to the 3D-mesh publishing, we want to map geomorphological structures of the cave interior.

Figure 3 presents the adopted tasks sequence. The inte- rior of the chamber was surveyed using a Terrestrial Laser Scanner to collect a point cloud data (see Section 2). To produce surface models, the point clouds collections was interpolated using the Poisson Surface Reconstruction (see Section 3). Surface models obtained present high level of detail (Figures 1 and 4) with millions of vertices and faces. For Web visualization with X3DOM, in real time, the surface model was simplified by a decimation process. The following paragraphs describe the decimation and the Web3D implementation. To be included in Web, surface models are converted to X3D format or other compatible.

Several technologies exist to create $3 \mathrm{D}$ content for the web. Some of them are plug-in based systems, that means they depend on an additional piece of software running inside a web browser. These technologies have some clear disadvantages such as, the distrust, security and incompatibility issues associated with the plug-in installation and the need to understand how the plug-in works to develop applications.

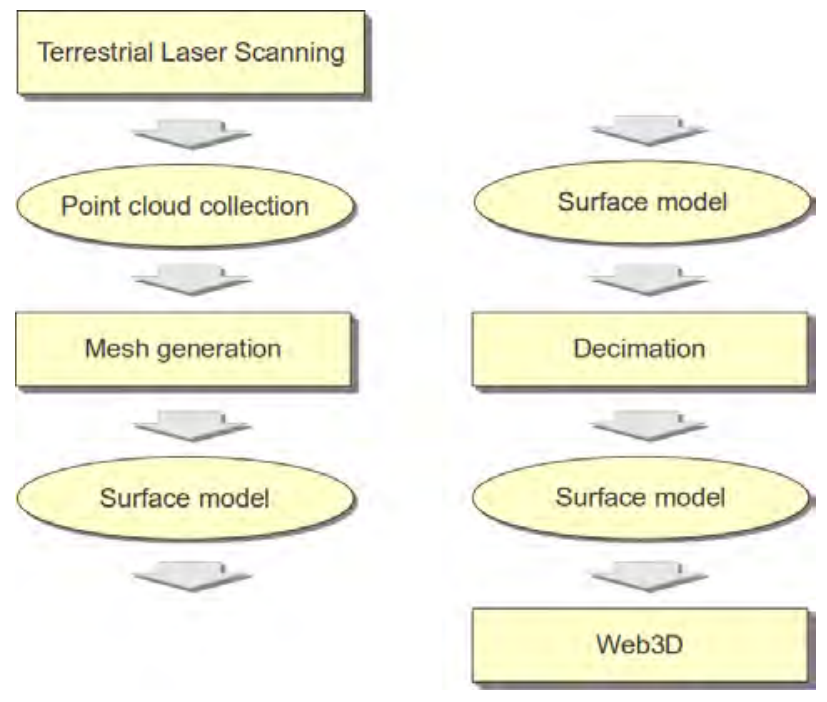

Figure 3: Tasks sequence from TLS surveying to 3D Web.

Nowadays, it is possible to integrate $3 \mathrm{D}$ content on the Web directly into the browser without plug-ins or additional components. This is the approach for the proposed framework presented in this paper that uses X3D, WebGL and $\mathrm{X} 3 \mathrm{DOM}$ enabling the 3D visualization and navigation in the interior of the Algar do Penico in the Firefox, Google Chrome, Safari and Opera web browsers without specific plug-ins. $\mathrm{X} 3 \mathrm{D}$ is used to represent the cave chamber $3 \mathrm{D}$ model and it is inserted on the user side for visualization in WebGL supported browsers with the X3D Document Object Model (X3DOM) technique. X3D is the ISO standard XML-based file format for representing 3D computer graphics. It is the successor of VRML, including a large 
number of new and extended features. A major difference between X3D and VRML is the use of XML as syntax. $\mathrm{X} 3 \mathrm{D}$ benefits in many ways of using XML since it is easy to read for both humans and computer systems, it is well supported, it is license free, the data is well structured and it is web compatible [6].

WebGL is an open standard software library for a lowlevel 3D graphics API based on OpenGL that generates interactive 2D and 3D graphics on any browser without installing additional plug-ins. WebGL uses HTML5 to include data content into the DOM (Document Object Model) and access to this content in the scene graph but HTML5 cannot do this directly with 3D data. In this context, the Web3D consortium has written recommendations about how to integrate HTML5 and 3D content, more specifically, Extensible 3D (X3D) [Mao 2011]. Although $\mathrm{X} 3 \mathrm{D}$ content is not supported by all web browsers directly right now, it is the trend that in the near future X3D elements will be able to be directly manipulated by the most common browsers with the DOM technique [7] [8].

$\mathrm{X} 3 \mathrm{DOM}$ is an open source framework with the objective of integrating HTML5 and X3D on top of WebGL[Behr et al. 2009]. X3DOM is a framework for integrating and manipulating (X3D) scenes as HTML5-DOM elements, which are rendered via WebGL and therefore does not require plug-ins for displaying the X3D content.

The 3D-mesh size is a problem for visualization efficiency and interactivity in real time on the Web. Models can present huge number of elements stored in large file size. Detailed and high-precision model can have 2.4GB (see Table 1). To produce a lighter model we decided to simplify the cave chamber 3D-mesh. One of the significant ways to simplify meshes is by geometry removal operations. These operations are called mesh decimation and consist in iteratively remove geometry unit such as vertices, edges or triangle faces [9].

For the 3D-mesh simplification we used the MeshLab multi-edge decimation function called Quadratic Edge Collapse Decimation. This function consists in removing the multi-edge mesh and the relative triangles and then connect the adjacent vertices to the new vertex [10].

In order to select a 3D-mesh with a reasonable size that do not compromise performance for Web publishing we tried to simplify the generated 3D-meshes presented in Table 1 (Section 3). The Quadratic Edge Collapse Decimation worked with the 3D-meshes generated from 10 and 11 octree depth. The 11 octree depth 3D-mesh was chosen due to its higher detail level, which refers to higher number and dimension of stalactite.

Three simplifications were generated from the 11 octree depth 3D-mesh. Table 2 presents the number of vertices, the number of triangle faces and the size of each simpli- fied 3D-mesh files in X3D format. These new simplified meshes were integrated with HTML5 using the InstantReality $^{4}$ framework. This framework provides tools for $3 \mathrm{D}$ data sets optimization. Tests of download time were performed in localhost environment. Waiting time varying between $7 \mathrm{~s}$ and $60 \mathrm{~s}$ were measured.

Table 2: Tests of download speed between three different mesh sizes.

\begin{tabular}{|c|c|c|c|}
\hline \# vertices & \# faces & $\begin{array}{c}\text { size } \\
\text { (MB) }\end{array}$ & $\begin{array}{c}\text { download } \\
\text { time (s) }\end{array}$ \\
\hline 500732 & 998856 & 60.2 & 60 \\
\hline 250791 & 499486 & 29.8 & 19 \\
\hline 125497 & 249696 & 14.6 & 7 \\
\hline
\end{tabular}

Considering the download times we selected the 3Dmesh with about 250 thousand faces and about 125 thousand vertices, as presented in Table 2. This 3D-mesh takes about 7 seconds to be ready for real time interaction in a Web browser.

Figure 4 illustrates the cave chamber 3D-mesh and its stalactites extremities represented by white dots. Lower tip of stalactites or local minima resulted from the algorithm 1 .

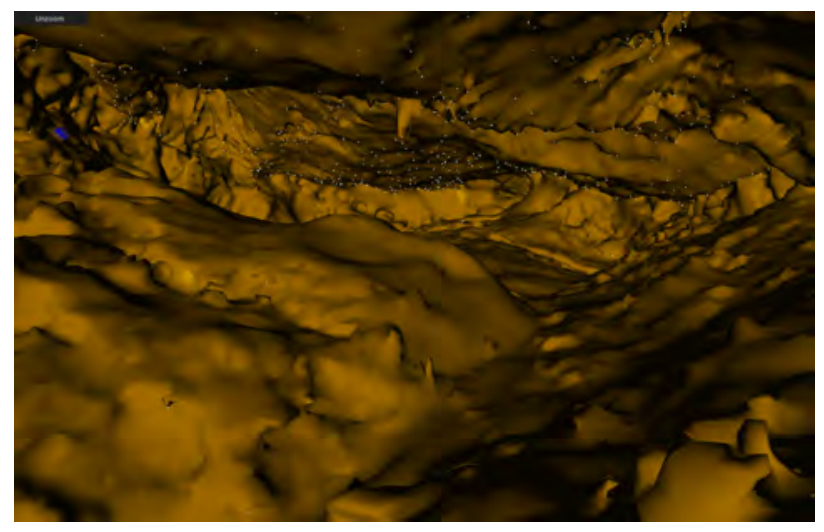

Figure 4: Web visualization of cave chamber 3D-mesh and local minima (stalactites extremities).

Figure 5 a) represents a 3D Web visualization of a particular area of interest. This area correspond to the biggest stalactite in the chamber. This visualization also contains the contour lines calculated for the same area. Figure $5 b$ ) shows the plane view of contour lines and local minima.

The developed framework for 3D visualization of the cave chamber is available on the Web at the address: http://193.136.227.170/sipclip/web3d/.

\footnotetext{
${ }^{4}$ http: //www.instantreality.org/
} 


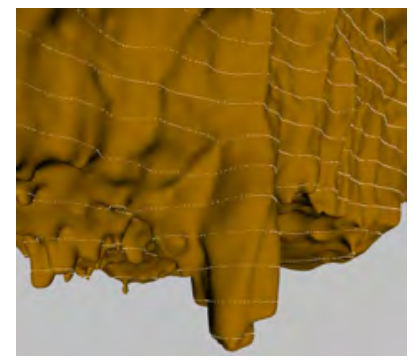

a)

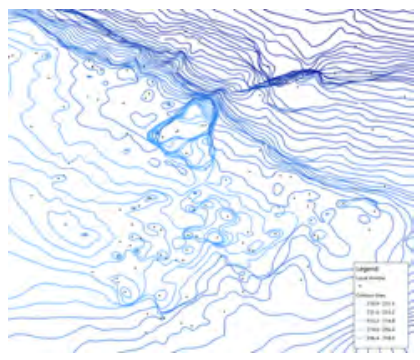

b)
Figure 5: 3D model (a) and respective plane view (b) of stalactite 3D-mesh and its contour lines.

\section{Conclusion}

This paper presented an approach for the 3D modeling and Web visualization of 3D data from a cave chamber. Chambers have many irregular surfaces and we obtained a point cloud with about 45 million points with high level of detail.

It was a challenge to work with such massive data collection to generate a surface model of the cave chamber to study its geomorphological features. For the 3D modeling process we used an open source tool called MeshLab. This software allowed to generate a 3D-mesh from the original point cloud with the goal to achieve a compromise between the complexity of the 3D-mesh, the realistic visualization and real time interaction in the chamber 3D model. For this purpose, we used the Poisson Surface Reconstruction method that generated a 3D-mesh with a about 5 million vertices and 10 million faces.

One of the main goals of this research project was to visualize and identify cave stalactites that could provide information on hidden cave features responsible for cave geomorphology. Stalactites extremities are local minima in the 3D-mesh. We described in this paper an algorithm to find local minima for the recognition of stalactites distribution, alignments and other properties of interest to the SIPCLIP project. It is also presented a topological structure for the 3D-mesh to get an efficient algorithm for the process of finding stalactites structures.

A framework for 3D visualization of cave chamber was made available on the Web using X3D, WebGL and X3DOM. Such solution does not require any additional plug-ins. The availability of Algar do Penico in a Web3D is interesting for the geospatial field. Researcher can navigate in this environment to visualize stalactites extremities and contour lines.

\section{Acknowledgments}

This work has been supported by the Portuguese government and EU - Funding, through the FCT - Fundação para a Ciência e Tecnologia funding of the SIPCLIP project
(PTDC/AAC-CLI/100916/2008).

\section{References}

[1] A. Roncat, Y. Dublyansky, C. Spotl, and P. Dorninger, "Full-3d surveying of caves: A case study of marchenhohle (austria)," in Proceedings of the International Association for Mathematical Geosciences (IAMG 2011), 2011.

[2] R. Tobler and S. Maierhofer, "A mesh data structure for rendering and subdivision," in International Conference in Central Europe on Computer Graphics, Visualization and Computer Vision, 2006, pp. 157-162.

[3] P. Cignoni, M. Callieri, M. Corsini, M. Dellepiane, F. Ganovelli, and G. Ranzuglia, "Meshlab: an opensource mesh processing tool." in Eurographics Italian Chapter Conference, V. Scarano, R. D. Chiara, and U. Erra, Eds. Eurographics, 2008, pp. 129-136.

[4] M. M. Kazhdan, M. Bolitho, and H. Hoppe, "Poisson surface reconstruction." in Symposium on Geometry Processing, ser. ACM International Conference Proceeding Series, A. Sheffer and K. Polthier, Eds., vol. 256. Eurographics Association, 2006, pp. 61-70.

[5] C. Shen, J. F. O'Brien, and J. R. Shewchuk, "Interpolating and approximating implicit surfaces from polygon soup," ACM Trans. Graph., vol. 23, no. 3, pp. 896-904, 2004.

[6] F. D. Cerbo, G. Dodero, and L. Papaleo, "Developing a web 3.0 e-learning portal with $\mathrm{x} 3 \mathrm{~d}$ technologies," in Eurographics Italian Chapter Conference, 2010, pp. 129-134.

[7] J. Behr, P. Eschler, Y. Jung, and M. Zöllner, "X3dom: a dom-based html5/x3d integration model." in Web3D, S. Spencer, D. Fellner, J. Behr, and K. Walczak, Eds. ACM, 2009, pp. 127-135. [Online]. Available: http://dblp.uni-trier.de/db/conf/ vrml/web3d2009.html\#BehrEJZ09

[8] I. Prieto, J. Izkara, and F. Delgado, "From point cloud to web 3d through citygml," in Virtual Systems and Multimedia (VSMM), 2012 18th International Conference on, 2012, pp. 405-412.

[9] P. S. Heckbert and M. Garland, "Optimal triangulation and quadric-based surface simplification," Computational Geometry, vol. 14, no. 1-3, pp. 49-65, 1999.

[10] H. Chen, X. Luo, and R. Ling, "Surface simplification using multi-edge mesh collapse," in Image and Graphics, 2007. ICIG 2007. Fourth International Conference on, 2007, pp. 954-959. 